\title{
Observational problems in determining the ages of open clusters
}

\author{
Elizabeth J. Jeffery \\ Department of Astronomy, The University of Texas at Austin, \\ Austin, TX 78712, USA \\ email: ejeffery@astro.as.utexas.edu
}

\begin{abstract}
Open clusters have long been objects of interest in astronomy. As a good approximation of essentially pure stellar populations, they have proved very useful for studies in a wide range of astrophysically interesting questions, including stellar evolution and atmospheres, the chemical and dynamical evolution of our Galaxy, and the structure of our Galaxy. Of fundamental importance to our understanding of open clusters is accurate determinations of cluster ages. Currently there are two main techniques for independently determining the ages of stellar populations: main sequence evolution theory (via cluster isochrones) and white dwarf cooling theory. We will provide an overview of these two methods, the current level of agreement between them, as well as a look to the current state of increasing precision in the determination of each. Particularly I will discuss the comprehensive data set collection that is being done by the WIYN Open Cluster Study, as well as a new Bayesian statistical technique that has been developed by our group and its applications in improving and determining white dwarf ages of open clusters. I will review the so-called bright white dwarf technique, a new way of measuring cluster ages with just the bright white dwarfs. I will discuss the first application of the Bayesian technique to the Hyades, also demonstrating the first successful application of the bright white dwarf technique. These results bring the white dwarf age of the Hyades into agreement with the main sequence turn off age for the first time.
\end{abstract}

Keywords. open clusters and associations: general, white dwarfs

\section{Introduction}

Age measurements are a fundamental problem in astronomy and essential for a number of astrophysically interesting problems. From the fundamental questions of the formation of the Universe to the creation of planets, knowing and understanding ages of astronomical objects is important. Answering the questions of when (for example, "When did the Universe form?" or "When did the halo/bulge/disk form in relation to each other?" or "When did the various elements form?" or "When do planets form?") is a crucial step in understanding the questions of how these phenomena occur.

Open star clusters have long been objects of interest in astronomy. They are a good approximation of essentially pure stellar populations and have proved useful for studies in a wide range of astrophysically interesting questions. They are useful in understanding stellar evolution and atmospheres, the chemical and dynamical evolution of our Galaxy, as well as its structure, and are essential to distance scale studies. In order to gain the most information from open clusters, accurate ages are essential.

Currently there are two main techniques for independently determining the ages of stellar populations: main sequence (MS) evolutionary theory (via cluster isochrones; e.g., Chaboyer et al. 1996) and white dwarf (WD) cooling theory (e.g., Winget et al. 1987). Open clusters provide the ideal environment for the calibration of these two important 
clocks, as well as providing the unique opportunity to test theory against theory and therefore increase our understanding of both.

Each of these two techniques is accompanied by both theoretical and observational obstacles to determining ages. In this paper I will focus mainly on the observational challenges in determining accurate cluster ages, as well as some of the current efforts to minimize these issues as best as can be currently done.

This paper is organized as follows: in Section 2 I will review the techniques for measuring MSTO and WD ages, and examine some of the major observational challenges in determining these ages accurately for open clusters. In Section 3 I will discuss a few solutions to alleviate some of the observational challenges discussed. These include improving cluster observations by collecting comprehensive data sets, such as is being done by the WIYN Open Cluster Study (WOCS) (Section 3.1); improving techniques for objectively measuring cluster ages, specifically discussing a new Bayesian statistical technique (Section 3.2); and finally, I will discuss a new technique to measure cluster WD ages, based on using the bright cluster WDs alone (Section 3.3). I will review the first application of the Bayesian technique and the bright WD technique to real data (the Hyades) by DeGennaro et al. (2009) and discuss those results in Section 4.

\section{Open cluster ages: Techniques and observational challenges}

As mentioned in Section 1, there are two main techniques used to measure open cluster ages: main sequence turn off (MSTO) ages and white dwarf (WD) cooling ages. I discuss each of these below.

\subsection{Main sequence turn off ages}

MSTO techniques are among the most mature methods for determining the ages of open clusters. A cluster's age is determined by fitting an isochrone to the MSTO on the color-magnitude diagram (CMD) (see, for example, Figure 1, taken from Sarajedini et al. 1999). Theoretical problems still exist in such models (as can be seen in Figure 1, for example, imperfect fits to the red giant branch (RGB), as well as different isochrones giving a best fit to the MSTO in different colors).

Some of the observational challenges in determining MSTO age include field stars and unresolved binaries, especially in the TO region. This gives a certain amount of width to MSTO (see Figure 1), and makes fitting isochrones by eye more difficult. Additionally, there are errors in the actual observations themselves, as well as errors in cluster distance, metallicity, and reddening (and, in some clusters, differential reddening; e.g., NGC 2477 (Hartnick et al. 1972). Because the position of a star on the CMD is affected by not only its color and magnitude, but also its distance, metallicity, and reddening, the effects of these properties can be confused for one another and can be difficult to disentangle, based on the CMD alone. A large uncertainty in one may cause an incorrect determination in another, and together may affect the best isochrone fit, and therefore the accuracy of the MSTO age.

\subsection{White dwarf cooling ages}

If an open cluster is sufficiently old, some of its members will be WDs. There is a simple relationship between a WD's luminosity and its cooling time (i.e., age) (Mestel, 1952). Cluster WDs show a low luminosity terminus whose location is determined by the age (Claver, 1995; von Hippel et al. 1995). I illustrate this in Figure 2; the figure shows a simulated CMD for a cluster of 3 Gyr. Over plotted are theoretical cooling tracks for WDs of several masses (ranging here from $0.5 M_{\odot}$ to $0.9 M_{\odot}$ ). WDs follow simple cooling 

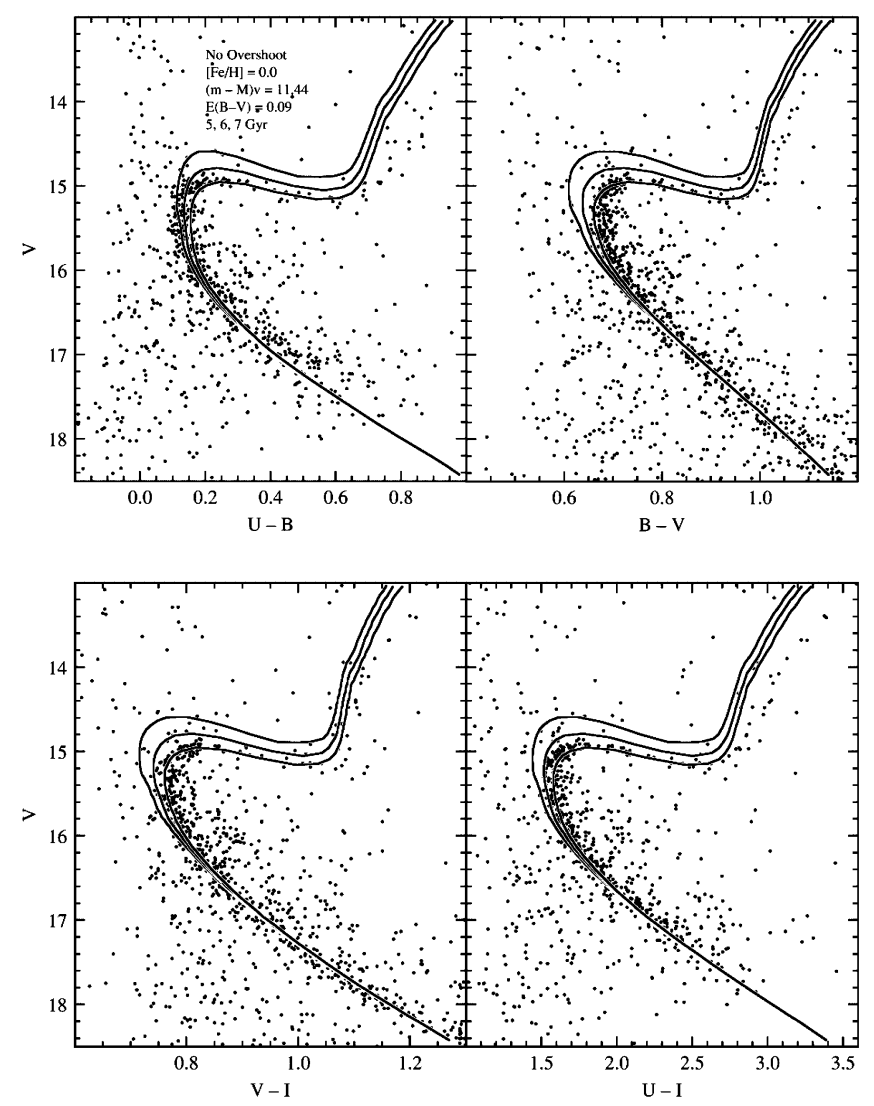

Figure 1. CMD for NGC 188, taken from Sarajedini et al. (1999), in several colors with theoretical isochrones overlaid. Note that no single isochrone gives a perfect fit in all colors, illustrating the difficulties that still exist in determining MSTO ages.

laws and therefore the rate at which a WD cools slows with time. This causes a "pile up" for WDs of different masses near the terminus of the WD sequence, causing the observed "hook" shape.

Like MSTO ages, the determination of cluster WD ages is also affected by observational errors, and errors in cluster distance and reddening. (They are less affected by errors in cluster metallicity.) Additional problems also include contamination from field stars (especially from field WDs) in the CMD and from background galaxies with similar colors. Cluster WDs are also intrinsically faint and therefore require large (or spacebased) telescopes to observe. In addition to these observational challenges, theoretical uncertainties still exist in the models for the coolest WDs, namely issues related to crystallization, phase separation (Isern et al. 2000; Metcalfe et al. 2004), and collisioninduced absorption (CIA) in the coolest WD atmospheres (Frommhold 1993; Kilic et al. 2006).

\subsection{Effect of observational errors on age}

As mentioned above, errors in cluster distance, metallicity, and reddening can affect the derived MSTO and WD age. For example, assuming no error in other parameters (observations, metallicity, and reddening) an error in the cluster distance modulus of 0.1 magnitudes translates to an error of $7 \%$ in the measured MSTO age. Similarly, a 


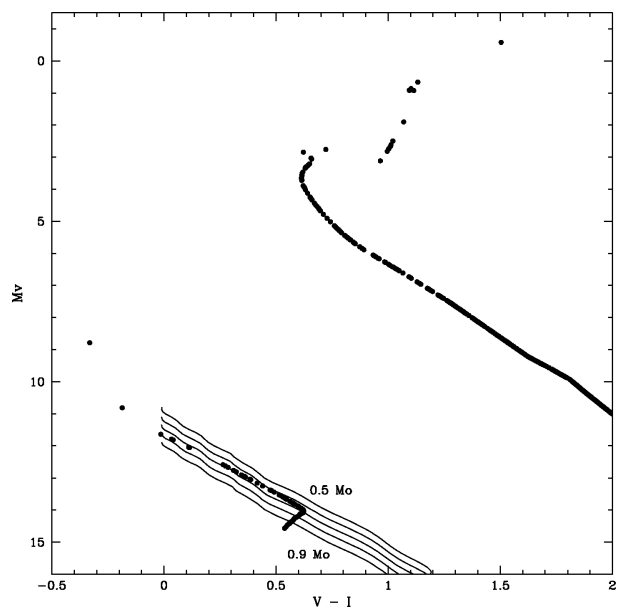

Figure 2. A simulated 3 Gyr open cluster. The figure illustrates where the WD cooling sequence falls on the CMD. The over plotted lines are cooling tracks for WDs of several masses. The hook shape of the WD terminus is explained by the "pile up" of WDs at the terminus.

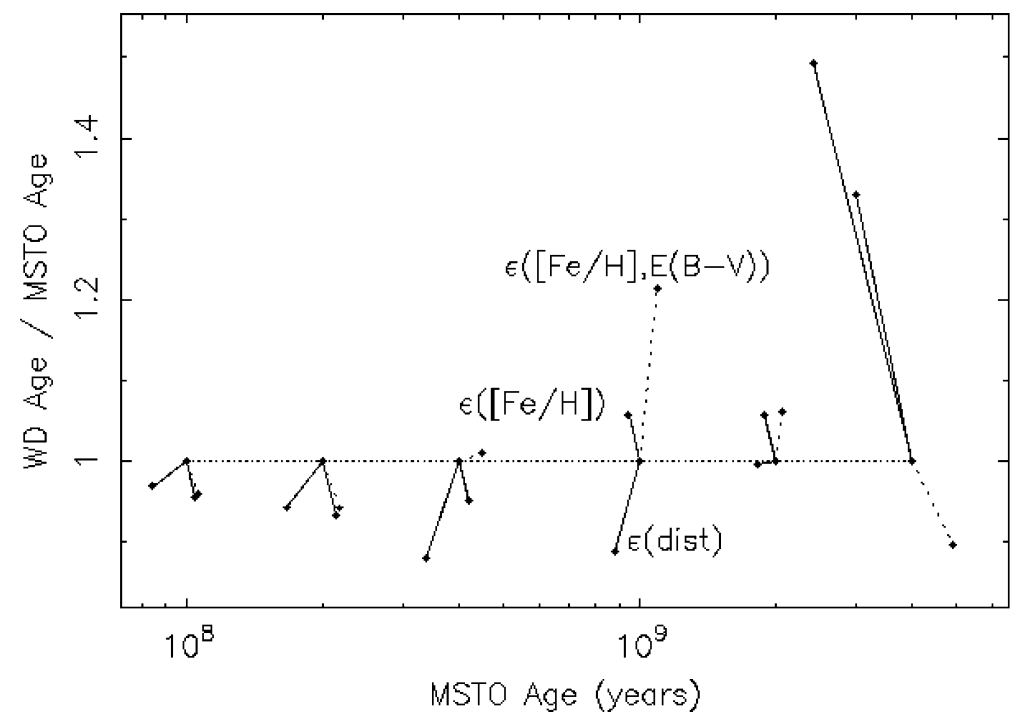

Figure 3. Effect of three types of errors on the MSTO and WD ages of open clusters, taken from von Hippel (2005). Thick lines show a distance overestimation of 0.2 magnitudes, the thin lines an overestimation of metallicity by $0.2 \mathrm{dex}$, and the dotted lines an overestimation of metallicity by 0.2 dex then compensated by a decrease in reddening enough to keep the MS at the same color.

distance modulus error of 0.2 and 0.5 magnitudes means an age error of $17 \%$ and $45 \%$, respectively.

This point was explored further by von Hippel (2005); Figure 3 is taken from that study. He illustrated how the WD and MSTO age are affected by errors in distance (thick solid line) of 0.2 magnitudes, metallicity (thin solid line) of 0.2 dex, and an error in metallicity of 0.2 dex then compensated for by decreasing the reddening to keep the MS at the same color (dotted line). 


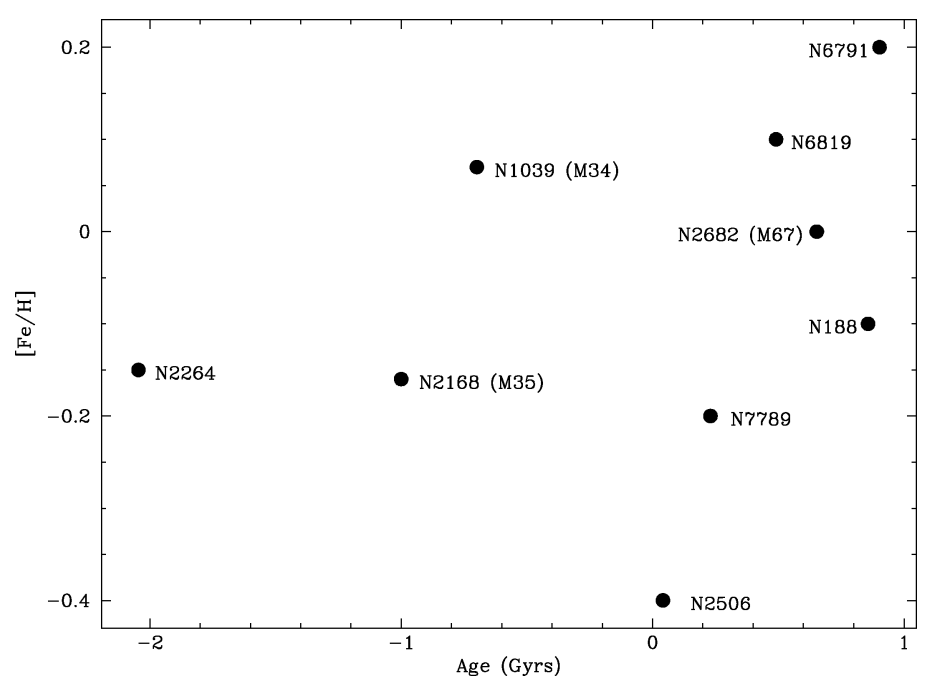

Figure 4. WOCS key clusters in age and metallicity space.

\subsection{Current agreement and calibration}

A calibration is needed to put these two chronometers on the same absolute scale. Open clusters provide the ideal environment for this calibration. This can be done by measuring the WD age and the MSTO age in a number of open clusters. Additionally, the WDs provide an independent check on MS models and the MS models check the WD models. This allows us to better understand the physics of each. Recent studies show good agreement up to 4 Gyr for open clusters (von Hippel, 2005).

\section{Challenges and solutions}

A few of the solutions to the observational challenges I have discussed in the previous section include collecting comprehensive data sets, improved statistical interpretations of CMDs, and a new technique to determine cluster WD ages. In the following sections, I examine each of these points.

\subsection{WOCS: A comprehensive study of open clusters}

Tools for the observational study of open clusters continue to improve and increase observational efficiency with (for example) mosaic CCDs and multi-object spectrographs. Photometric, spectroscopic, and astrometric measurements can be made with higher precision than before. The WIYN Open Cluster Study (WOCS) is a collaboration formed with the goal to create a comprehensive and definitive photometric, spectroscopic, and astrometric database for several key clusters (see Figure 4). Such a comprehensive and complete data set allows for the investigation of critical astrophysical problems through the study of open clusters.

Efforts are being done by WOCS on nearly all fronts of data acquisition. These efforts include: absolute photometry to obtain carefully calibrated CMDs (e.g., Sarajedini et al. 1999); monitoring of relative photometry to find cluster variable stars (e.g., Kafka \& Honeycutt 2003); spectroscopic observations to determine metallicities and elemental abundances, including lithium (e.g., Steinhauer \& Deliyannis 2004); radial velocity 
studies as a means of finding binary stars and studying cluster dynamics (e.g., Geller et al. 2008); and astrometry to measure proper motions, separating cluster members from contaminating field stars (e.g., Platais et al. 2003). When used together, the information gleaned from these multiple studies provide valuable insights to many astrophysical problems through the study of open clusters.

\subsection{Measuring ages using Bayesian statistics}

Despite many high quality datasets having been collected on open clusters, age precision of better than $10-20 \%$ is still generally out of reach. The greatest gains that can currently be made in age precision will require improved modeling techniques (see also work done by Tosi et al. 1991, Tosi et al. 2007; Hernandez \& Valls-Gabaud 2008).

A new technique has been developed to determine cluster ages using Bayesian statistics (see von Hippel et al. 2006; Jeffery et al. 2007; and DeGennaro et al. 2009, and in the poster at this conference by DeGennaro et al.). Briefly, the Bayesian technique derives a posterior probability distribution for a cluster's age, metallicity, distance, and line-ofsight absorption by objectively incorporating our prior knowledge of stellar evolution, star cluster properties, and data quality estimates. It incorporates a Miller \& Scalo (1979) initial mass function, MS and giant branch stellar evolution time scales of Girardi et al. (2000), the initial-final mass relation (IFMR) from Weidemann (2000), WD cooling timescales of Wood (1992), and WD atmosphere colors from Bergeron et al. (1995). Two additional sets of MS models have also been added - Yale-Yonsei (Yi et al. 2001), and a finer grid of models from the Dartmouth Stellar Evolution Database (DSED, Dotter et al. 2008), as well as updated versions of Bergeron WD atmosphere models.

\subsection{The bright white dwarf technique}

Jeffery et al. (2007) demonstrated the theoretical feasibility of determining WD ages of clusters using the brighter WDs alone. Briefly, this technique relies on the subtle differences in slope and position of the WD cooling sequence relative to the MS for clusters of different ages (as illustrated in Figure 5).

For an individual cluster WD, its total age (i.e., the age of the cluster) is its WD cooling time plus the lifetime of its MS counterpart. The cooler WDs are more massive and hence evolved off the MS more quickly; i.e., they have spent a larger fraction of their total lifetime as WDs. In these cases, their WD cooling time is good measure of the total cluster age. I have plotted the relationship between the ratio of a WD's cooling time and the total cluster age as a function of absolute magnitude in Figure 6.

The bright WD technique assumes that the IFMR is universal and single-valued, which is the general consensus among researchers (Weidemann 2000). Because of this dependence on the IFMR, this technique is a relative age indicator and requires calibration. As will be discussed in the next section, recent work on the Hyades is the first step in such a calibration.

\section{The white dwarf age of the Hyades}

The Hyades is one of the most well-studied open clusters in the sky. Perryman et al. (1998) report a MSTO age for this cluster of $625 \pm 50 \mathrm{Myr}$ and a distance to the center of the cluster (based on trigonometric parallaxes from Hipparcos) of $m-M=3.33 \pm 0.01$. High resolution spectroscopy has been used to determine the metallicity to high accuracy, $[\mathrm{Fe} / \mathrm{H}]=+0.103 \pm 0.008$ (Taylor \& Joner 2005, based on their re-analysis of Paulson et al. 2003). In order to apply the Bayesian technique to real data for the first time, this cluster was the logical choice. 


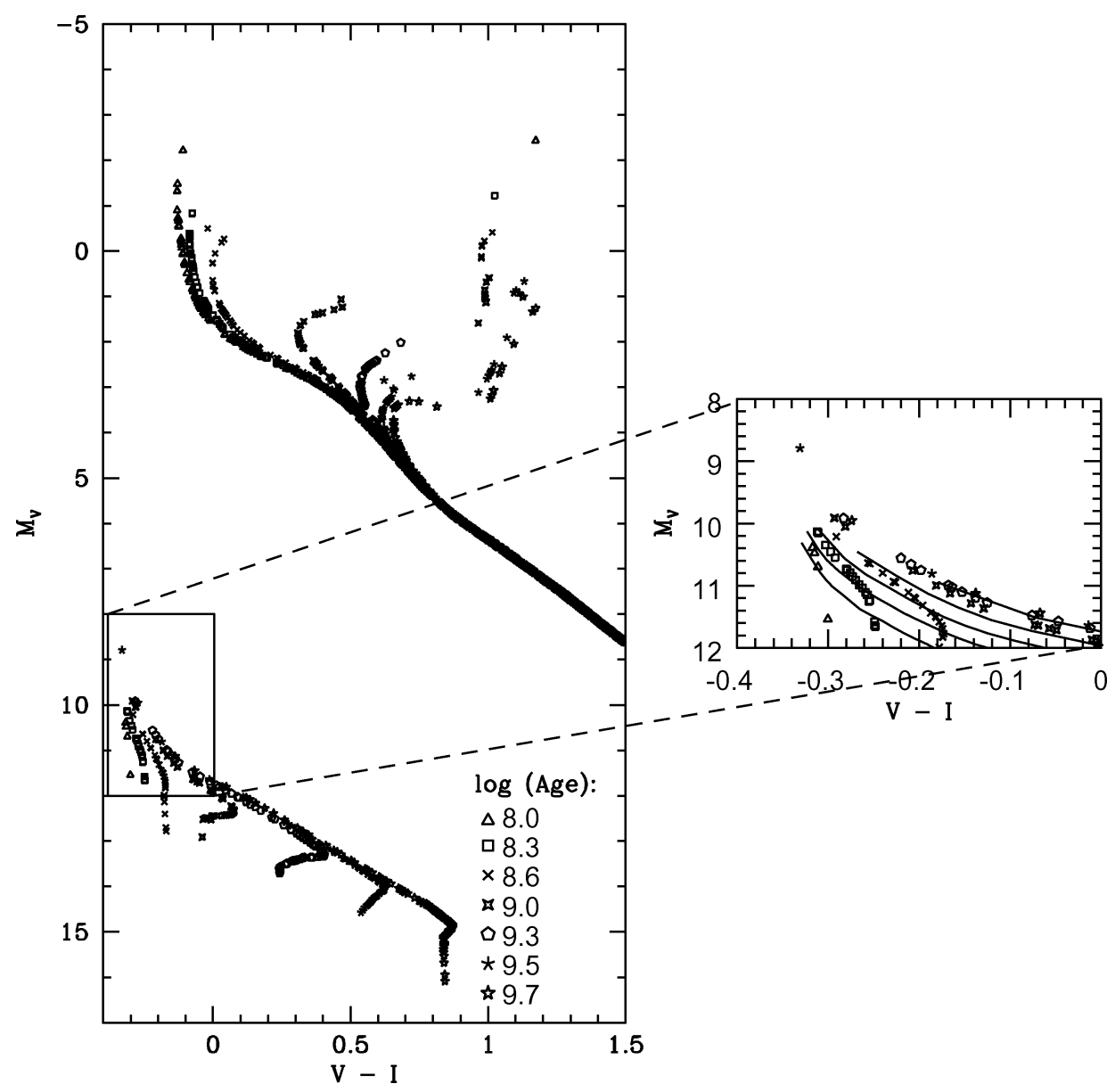

Figure 5. Simulated clusters for several different ages. The expanded region shows the regime of the brighter WDs, clearly showing the subtle differences in the slopes and positions of the WD cooling sequences relative to the MS for clusters of different ages. This makes it possible to extract age information without observing the faintest WDs.

Previous studies to determine the WD age of the Hyades cluster have produced a result (300 Myr; Weidemann 1992) that is about half the measured MSTO age (625 Myr; Perryman et al. 1998). Weidemann (1992) suggested that this discrepancy is due to the dynamical evaporation of stars from this cluster; the coolest WDs are no longer present. This is illustrated in Figure 7. This is the CMD for the WD region of the Hyades with a WD isochrone for the MSTO age of the cluster over plotted, demonstrating the lack of cool WDs that are expected to populate the bottom of the WD cooling sequence. In the absence of any data on these missing faint WDs, traditional techniques to determine WD ages can provide at best a lower limit to the WD age. (In reference to this figure and the results, however, it should be noted that when doing a best fit of the data, the Bayesian technique fits the data (UBV photometry was used for this study) in magnitudemagnitude space, not color-magnitude space, as is plotted in Figure 7.)

As summarized earlier, in Jeffery et al. (2007) showed the possibility of determining cluster WD ages from just the bright WDs, when the coolest WDs are not observed. 


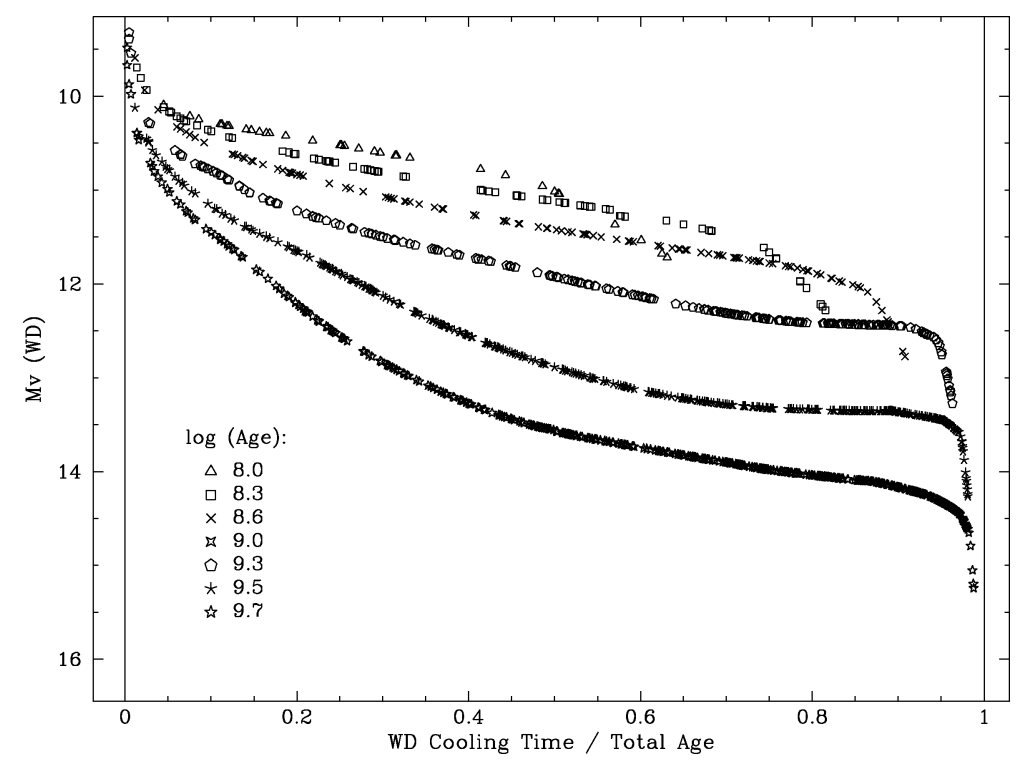

Figure 6. The relationship between the ratio of an individual WD's cooling time and the total cluster age and the absolute magnitude of that WD.

Because the coolest WDs are missing from the Hyades, the bright WD technique is required to measure the true WD age (rather than a lower limit, as was done previously).

DeGennaro et al. (2009) applied the Bayesian technique to the Hyades, the first application of the technique to real data, and has proven the technique successful. This is also the first empirical evidence that the bright WD technique yields reasonable and precise ages for real data, as well as providing an important step in calibrating the technique. (See Figure 8.)

\section{Conclusions}

Age is a quantity of importance to all areas of astrophysics. The study of open clusters, as nearly pure stellar populations, continues to be an important area of study in stellar astronomy. They provide the environment for answering many questions of astrophysical import and measuring accurate ages for open clusters is essential to fully leveraging this potential.

MSTO and WD cooling ages are the two main techniques used to determine ages of these stellar populations. The CMD is the primary tool for these age determinations. Observational challenges for each method include observational errors, as well as errors in cluster parameters (that is, distance, metallicity, and reddening). Challenges to fitting MSTO isochrones come as a result of field star contamination and unresolved cluster binaries. For WDs, there is also the issue of contamination from field stars (especially field WDs in the WD region) and background galaxies. Also, cluster WDs are faint, thus requiring large or space-based telescopes to observe them.

These problems can be alleviated in part by comprehensive data sets, such as are being assembled by WOCS. WOCS is creating a comprehensive and definitive photometric, spectroscopic, and astrometric database for fundamental clusters, covering a large range 


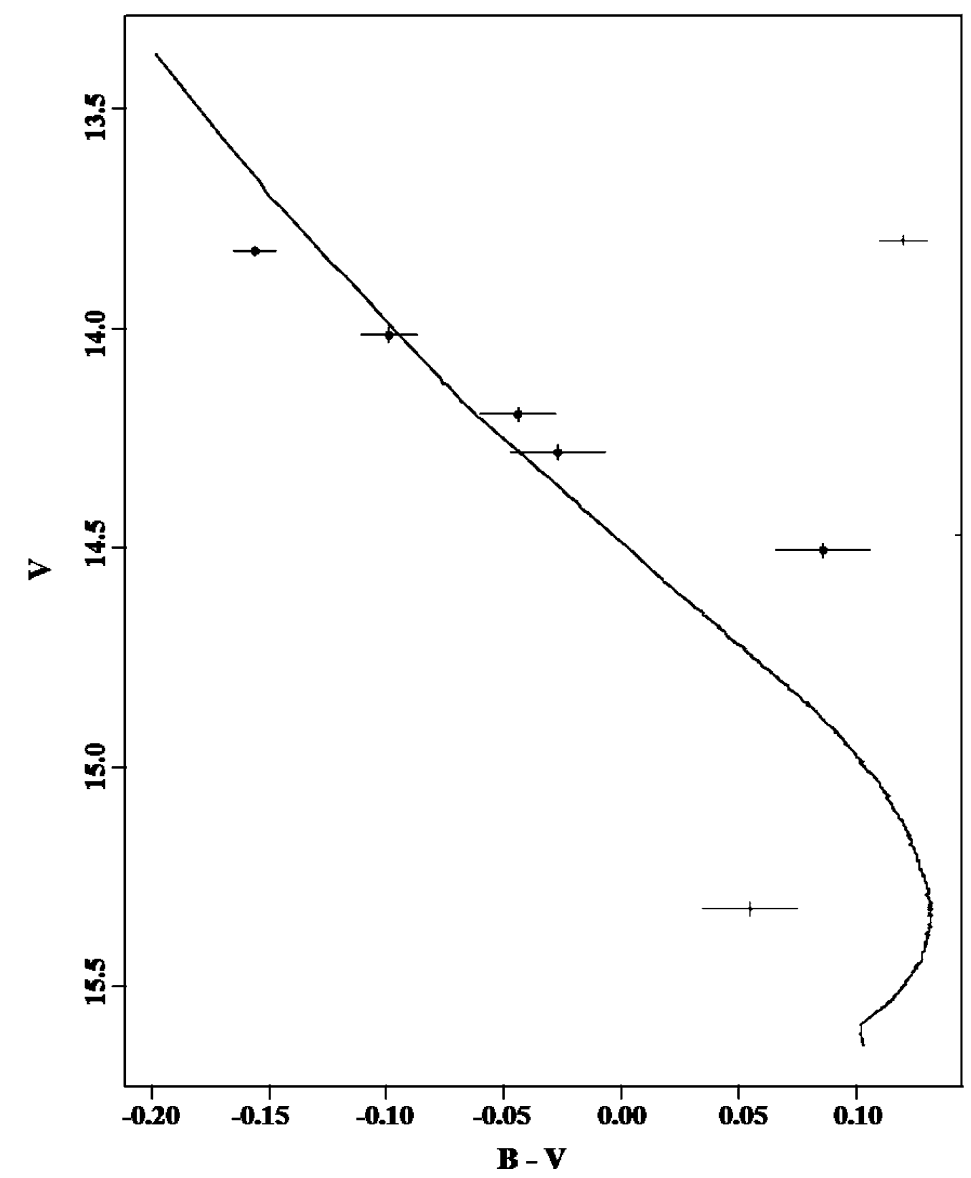

Figure 7. CMD of the WD region of the Hyades. The over plotted WD isochrone is for an age equal to the MSTO age. This demonstrates the lack of cool WDs that are expected to populate the bottom of the WD cooling sequence. However, note that the Bayesian technique does a best fit to the data based on magnitude-magnitude plots, not CMDs. In this analysis, UBV colors were used.

of ages and metallicities. Many critical astrophysical problems are being addressed as a result of the data being gathered.

We are continuing an effort to calibrate WD and MSTO ages using open clusters. In order to improve accuracy of the measured ages, our group has developed a technique using Bayesian statistics. This technique is proving successful in determining cluster WD ages to higher precision than before.

Additionally, here I have reviewed the so-called bright WD technique, a new technique to determine cluster WD ages using just the bright WDs (i.e., not observing the faintest/coolest cluster WDs). DeGennaro et al. (2009) have demonstrated the technique by applying it on the Hyades as a test case, whose cool WDs are missing due to the dynamical evaporation of the cluster. These results bring the WD age of the Hyades in agreement with the MSTO age for the first time, as well as provide a first step in calibrating the bright WD technique. (For further discussion of this result, see the poster by DeGennaro et al. at this conference.) 


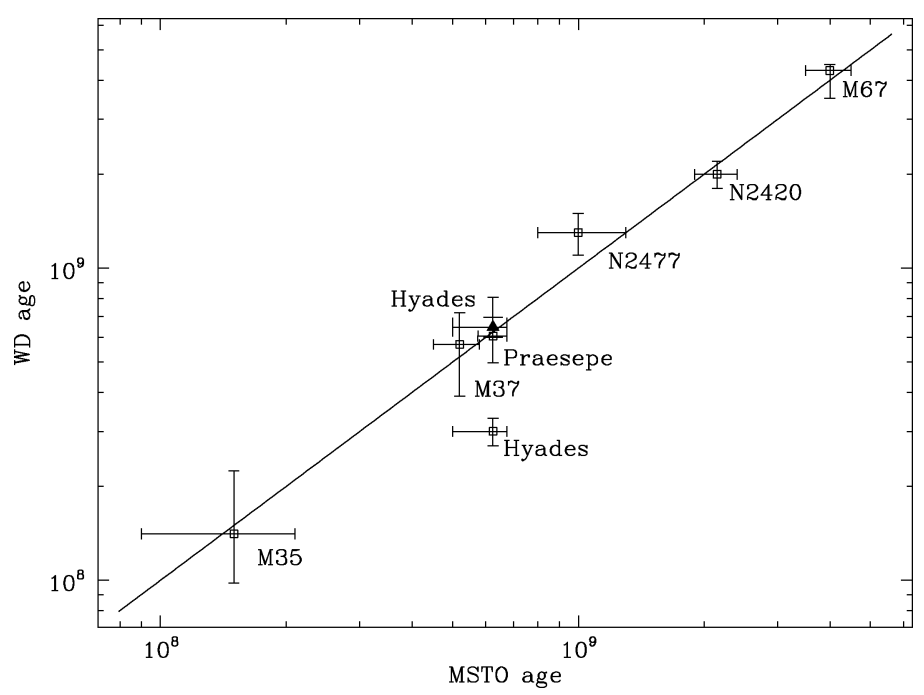

Figure 8. WD versus MSTO age for seven clusters, adapted and updated from von Hippel (2005). The age derived from the WDs in the Hyades by DeGennaro et al. (2009) using the bright WD technique brings the WD age of the Hyades into agreement with the MSTO age for the first time (solid triangle). The solid line shows the one-to-one correspondence between the WD and MSTO ages, and the gray point shows the most reliable WD age of the Hyades prior to this work (Weidemann 1992).

\section{Acknowledgments}

This material is based upon work supported by the National Aeronautics and Space Administration under Grant No. NAG5-13070 issued through the Office of Space Science.

\section{References}

Bergeron, P., Wesemael, F., \& Beauchamp, A. 1995, pasp, 107, 1047

Chaboyer, B., Demarque, P., \& Sarajedini, A. 1996, ApJ, 459, 558

Claver, C. F. 1995, PhD. Thesis, The University of Texas at Austin

DeGennaro, S., von Hippel, T., Jefferys, W. H., Stein, N., van Dyk, D., \& Jeffery, E. J., ApJ, in press

Dotter, A., Chaboyer, B., Jevremovic, D., Kostov, V., Baron, E., \& Ferguson, J. W. 2008, ApJS, in press, arXiv:0804.4473

Frommhold, L. 1993, Collision-Induced Absorption in Gases (Cambridge: Cambridge Univ. Press)

Geller, A. M., Mathieu, R. D., Harris, H. C., \& McClure, R. D. 2008, AJ, 135, 2264

Girardi, L., Bressan, A., Bertelli, G., \& Chiosi, C. 2000, A\&AS, 141, 371

Hartnick, F. D. A., Hesser, J. E., \& McClure, R. D. 1972, ApJ, 174, 554

Hernandez, X. \& Valls-Gabaud, D. 2008, MNRAS, 383, 1603

Isern, J., Garca-Berro, E., Hernanz, M., \& Chabrier, G. 2000, ApJ, 528, 397

Jeffery, E. J., von Hippel, T., Jefferys, W. H., Winget, D. E., Stein, N., \& DeGennaro, S. 2007, ApJ, 658, 391

Kafka, S. \& Honeycutt, R. K. 2003, AJ, 126, 276

Kilic, M., von Hippel, T., Mullally, F., Reach, W. T., Kuchner, M. J., Winget, D. E., \& Burrows, A. $2006, A p J, 642,1051$

Mestel, L. 1952, MNRAS, 112, 583 
Metcalfe, T. S., Montgomery, M. H., \& Kanaan, A. 2004, ApJ, 605, L133

Miller, G. E. \& Scalo, J. M. 1979, ApJS, 41, 513

Paulson, D. B., Sneden, C., \& Cochran, W. D. 2003, AJ, 125, 3185

Perryman, M. A. C. et al. 1998, A\& A, 331, 81

Platais, I., Kozhurina-Platais, V., Mathieu, R. D., Girard, T. M., \& van Altena, W. F. 2003, AJ, 126, 2922

Sarajedini, A., von Hippel, T., Kozhurina-Platais, V., \& Demarque, P. 1999, AJ, 118, 2894

Steinhauer, A. \& Deliyannis, C. P. 2004, ApJ, 614, 65

Taylor, B. J. \& Joner, M. D. 2005, ApJS, 159, 100

Tosi, M., Greggio, L., Marconi, G., \& Focardi, P. 1991 AJ, 102, 951

Tosi, M., Bragaglia, A., \& Cignoni, M. 2007, MNRAS, 378, 730

von Hippel, T., Gilmore, G., \& Jones, D. H. P. 1995, MNRAS, 273, L39

von Hippel, T. 2005, ApJ, 622, 565

von Hippel, T., Jefferys, W. H., Scott, J., Stein, N., Winget, D. E., DeGennaro, S., Dam, A., \& Jeffery, E. J. 2006, ApJ, 645, 1436

Winget, D. E., Hansen, C. J., Liebert, J., van Horn, H. M., Fontaine, G., Nather, R. E., Kepler, S. O., \& Lamb, D. Q. 1987, ApJ, 315, L77

Weidemann, V., Jordan, S., Iben, I. J., \& Casertano, S. 1992, AJ, 104, 1876

Weidemann, V. 2000, $A \mathscr{E} A, 363,647$

Wood, M. A. 1992, ApJ, 386, 539

Yi, S., Demarque, P., Kim, Y.-C., Lee, Y.-W., Ree, C. H., Lejeune, T., \& Barnes, S. 2001, ApJS, 136,417

\section{Discussion}

M. Tosi: Could you explain how the applications of the Bayesian method has allowed to reconcile the Hyades age taking evaporation into account? What is exactly that brings the new age up on the curve fitting all the other clusters?

E. Jeffrey: The Bayesian technique measures the age of the Hyades by fitting the white dwarfs that are available - that is, the bright (hot) white dwarfs. We've shown that it is theoretically possible to do this, and the case of the Hyades shows that this technique works on real data. The age is determined by doing a best fit to the white dwarf sequence (in $U B V$ ), which exploits the slope of the white dwarf sequence and its relative distance from the main sequence.

R. JEFFRIES: How can the age estimates or errors in age estimates from the WD cooling tracks (for the Hyades in particular) be believed when the "best fit" model in $V$ vs. $(B-V)$ is clearly a poor fit?

E. JEFFERY: The plot shown here $(V$ vs. $(B-V))$ is just for illustrative purposes, since most of us are used to thinking in the color-magnitude plane. However, there are a couple of things to note: The first is that $B V$ data is not all that was used to fit and measure this age; we also had $U$ data. Second is that the Bayesian algorithm fits the data in the magnitude-magnitude plane (that is, $U$ vs. $B, B$ vs. $V$, etc.), not the color-magnitude plane. Taking all three filters into account, the age we fit is the best fit. (The poster by DeGennaro, as well as his recently submitted paper explaining this result, expounds on this further.) Also, the Hyades has a problem that most open clusters do not, that is, the depth of the cluster (about 10\% of the distance) can affect individual stars, making them appear brighter or fainter than we'd expect (therefore affecting their position slightly in the color- magnitude plane, making some appear slightly off the isochrone in one particular color, as seen here). 
A. WEST: How much of your age uncertainty is due to uncertainty in the WD progenitor lifetimes?

E. JeFFERY: Uncertainty in cluster ages from the cooling times of the coolest white dwarfs depends little on their progenitor lifetimes. This is because the coolest white dwarfs came from the massive stars whose progenitor lifetimes are small compared to their cooling times. Therefore we can take their cooling time to be the cluster age. For the hot (bright) white dwarfs, the cooling age of an individual star is a smaller fraction of its total age (i.e., the cluster age). However, because we are fitting multiple white dwarfs along the white dwarf sequence (rather than an individual white dwarf), we don't need to worry about progenitor lifetimes. Although, that said, the bright white dwarf technique assumes a universal and single-valued initial-final mass relation, and requires calibration. Our Hyades results presented here are the first step in that calibration.

H. RICHER: Have you tried your technique on the bright end of the white dwarf cooling sequence in the two globular clusters with lots of white dwarfs?

E. JEFFERY: We have not yet, but we hope to in the future. However, the technique hasn't been theoretically tested at ages that great. It receives leverage from the mass spread in the upper white dwarf sequence and there is not much of a mass spread in clusters of globular cluster age. But we are interested in testing it.

G. De Marchi: You said that the coolest WDs in the Hyades are no longer there because they evaporated. But dynamical evaporation should start from the bottom of the main sequence, so say $0.3 M_{\odot}$ stars should have long ago evaporated from the Hyades well before WDs, but they are still there?

E. JEFFERY: That's an interesting dynamical question. I'm not sure why it is that way.

I. KING: You cited the slope of the brighter part of the WD sequence as one method of age dating, and statistical fitting of theoretical isochrones as another. But you showed a slide in which the stars had a quite different slope from that of the isochrone. How do you explain this discrepancy?

E. JEFFERY: Just to clarify, we fit isochrones to the slope of the cooling sequence. (That is, they are not different methods; the different method comes from fitting the entire cooling sequence vs. fitting just the bright portion.) As for why the isochrone appears to be a poor fit to the data in the $V$ vs. $B-V$ color-magnitude diagram, I refer my answer to Rob Jeffries's question. 